\title{
Diagnóstico de gestão do conhecimento: o caso de uma organização de pesquisa e desenvolvimento
}

\author{
DerisedeCufa Doutoranda em Engenharia e Gestão do Conhecimento. Universidade Federal de Santa Catarina (UFSC) - \\ Brasil. denise cuffa@hotmail.com \\ Rajanecosa Doutoranda em Engenharia e Gestão do Conhecimento. Faculdade CESUSC(CESUSC) - Brasil. \\ rejanecostafloripa@gmail.com \\ Cevesan Tabajaa Vama Doutorando em Engenharia e Gestão do Conhecimento. Instituto Federal de Santa Catarina (IFSC) - Brasil. \\ tabajara@ifsc.edu.br \\ Darzdi SaleteTrzaiak Doutor em Engenharia de Produção. Instituto Euvaldo Lodi de Santa Catarina - Brasil. dorzeli@gmail.com \\ FemendbOsturi Guthier Doutor em Engenharia de Produção. Universidade Federal de Santa Catarina (UFSC) - Brasil. \\ gauthier@egc.ufsc.br
}

\section{RESUMO}

O conhecimento é reconhecido como um importante recurso para que as organizações se tornem competitivas em seu mercado de atuação. A gestão desse recurso consiste em uma prática adotada pelas organizações para a busca de eficiência e efetividade dos seus processos. Essa pesquisa realizou um diagnóstico de gestão do conhecimento em uma organização de pesquisa e desenvolvimento de Florianópolis. É uma pesquisa de natureza aplicada, com abordagem qualitativa e descritiva. Foi aplicado o modelo de diagnóstico de gestão do conhecimento proposto por Bukowitz e Williams (2002) a cinco dirigentes de uma organização de pesquisa e desenvolvimento, intensiva em conhecimento. Os resultados demonstram que a gestão do conhecimento da organização apresentou baixa avaliação, a partir da percepção dos dirigentes pesquisados. A maior parte dos resultados abaixo da média encontra-se em seções pertencentes ao nível tático do processo de gestão do conhecimento (Obtenha, Aprenda e Contribua). Apenas a Seção Utilize foi melhor avaliada pelos dirigentes. No nível estratégico, destaca-se a Seção Avalie com o menor resultado e a Seção Construa/Mantenha como a seção melhor avaliada. Por meio do diagnóstico realizado foi elaborado um programa de ações visando a melhoria das seções que apresentaram baixa avaliação.

Palavras-chave: Gestão do conhecimento. Diagnóstico de gestão do conhecimento. Organização intensiva em conhecimento.

\section{Knowledge management diagnosis: the case of a research and development organization}

\begin{abstract}
Knowledge is recognized as an important resource for organizations to become competitive in their market. The management of this resource consists of a practice ad opted by organizations that search for efficiency and effectiveness of their processes. This research carried out a knowledge management diagnosis in a research and development organization in Florianópolis. It is an applied research, with a qualitative and descriptive approach. Knowledge management diagnostic model proposed by Bukowitz and Williams (2002) was applied to five leaders of a knowledge-intensive research and development organization. The findings showed that knowledge management of the organization presented low evaluation in the perception of the managers. Most below-average results are found in sections of tacit level of knowledge management process (Get, Learn and Contribute). Only the Use section was better jud ged by leaders. At strategic level, we highlight the Evaluate section with the lowest result and the Build/Hold section as the best evaluated section. Due to this diagnosis, an action program was developed aiming at improvement in those sections with low evaluation.

Keywords: Knowledge management. Knowledge management diagnosis. Knowledge-intensive organization.
\end{abstract}




\section{INTRODUÇÃO}

Na última década, o conhecimento passou a ser melhor percebido pelas organizações como um recurso que oportuniza vantagem competitiva frente ao mercado, tendo em vista que este recurso é difícil de negociar e imitar (FLEURY; OLIVEIRA JR, 2001). A gestão do conhecimento (GC) é um recurso que vem ganhando força por viabilizar a interação com o fluxo de informações existentes nas organizações (VASCONCELOS; CÂNDIDO, 2008). Quando se trata de organizações intensivas em conhecimento, a gestão do conhecimento passa a ser uma necessidade, pois as mesmas têm esse recurso como produto. Este é reconhecido como um atributo essencial para as mesmas (NADAl, 2006). As organizações de pesquisa e desenvolvimento (P\&D) são exemplos de ambientes intensivos de criação de conhecimento (MADHOK, 1996; LANGLOIS, 2003).

No contexto da GC, o fator humano - capital intelectual - é importante, e a atuação das lideranças e chefias é fator decisivo para determinar os caminhos que a organização deve seguir. Os ambientes e as práticas existentes são elementos importantes para a GC (GARCIA; COLTRE, 2017). Gerir o conhecimento é um desafio permanente para as organizações, pois esse recurso não se limita à temporalidade, espaço geográfico, e encontra-se nos indivíduos e em suas lideranças. Os modelos de GC são importantes para as organizações dado que os mesmos tratam o conhecimento enquanto recurso estratégico que oportuniza a competitividade das mesmas (JANUZZI; FALSARELLA; SUGAHARA, 2016). No entanto, muitas organizações não dispõem de ferramentas adequadas para que ocorra a obtenção, o uso, a aprendizagem, o compartilhamento, a avaliação e o descarte do conhecimento (BRITO; OLIVEIRA; CASTRO, 2012). 0 modelo de Bukowitz e Williams propõe uma metodologia que pode ser aplicável para avaliar a GC em organizações, por meio de seus aspectos operacionais e estratégicos. Essa pesquisa teve como objetivo realizar um diagnóstico de gestão do conhecimento em uma organização de pesquisa e desenvolvimento, intensiva em conhecimento.

A pesquisa está estruturada em seis seções. A primeira refere-se à introdução, que contextualiza 0 tema pesquisado, sua relevância e o objetivo de pesquisa. Na segunda seção é apresentada a fundamentação teórica composta por conceitos basilares da GC e do modelo de Diagnóstico de Gestão do Conhecimento de Bukowitz e Williams (2002). Na terceira seção são descritos os procedimentos metodológicos utilizados para a realização da pesquisa. Na quarta seção são apresentados e discutidos os resultados encontrados e, proposto um programa de ações de gestão do conhecimento. Finalmente, a sexta seção apresenta as considerações finais.

\section{FUNDAMENTAÇÃO TEÓRICA}

A seguir são apresentados a definição de gestão do conhecimento e seus desafios e, em seguida, é descrito o modelo de gestão do conhecimento utilizado para a realização desta pesquisa.

\subsection{A gestão do conhecimento e seus desafios}

A gestão do conhecimento tem sido bastante estudada, em decorrência do desafio advindo do surgimento das novas tecnologias (SOUZA; KURTZ, 2014) e por consistir em uma prática adotada pelas organizações para a busca de eficiência e efetividade dos seus processos (OLIVA, 2014). É uma área de conhecimento que possui diversos conceitos, perspectivas e abordagens (SCHOLL et al., 2004).

Dentre as definições de gestão do conhecimento está que esta consiste em um processo estruturado em que "[...] todos os elementos (seções) do processo devem ser geridos em relação uns aos outros para alcançar a mistura e a quantidade corretas de conhecimento e a capacidade de distribuí-los" (BUKOWITZ; WILLIAMS, 2002, p. 24-25). 0 objetivo desse processo de GC consiste em "[...] identificar, desenvolver, disseminar e atualizar o conhecimento estratégico relevante para a empresa, seja por meio de processos internos, seja por meio de processos externos" (FLEURY; OLIVEIRA JR, 2001, p. 19), o que pode refletir no alcance dos objetivos organizacionais (DAVENPORT; PRUSAK, 1998). 
Alguns desafios críticos da gestão do conhecimento consistem em gerenciar com efetividade 0 conteúdo, facilitar a colaboração, ajudar os trabalhadores do conhecimento a se conectarem e a encontrarem especialistas, e ajudar a organização a aprender e tomar decisões baseadas tanto em dados e informações completas e válidas quanto em conhecimento (DALKIR, 2005). Técnicas de gestão do conhecimento possibilitam a formalização do conhecimento dos indivíduos (conhecimento tácito) por meio de procedimentos escritos ou de outros registros (imagens e som, por exemplo). Esse processo de formalização possibilita que a organização obtenha como resultado a melhoria contínua de seus processos (OLIVA, 2014).

Os "contratos psicológicos" também são considerados um dos desafios da GC. Os mesmos são baseados em lealdade emocional, hierarquias, incentivos e habilidades, e motivação da equipe (FOSS, 2007). A solução desses problemas pode ocorrer por meio da atuação no microambiente individual e da realização do exercício de dimensionar as "transações de conhecimento" (FOSS, 2002, p. 16), tal como se dimensionam os custos de uma transação operacional já conhecida e que podem determinar o risco organizacional (FOSS, 2002).

Finalmente, a gestão do conhecimento também tem como desafio diagnosticar e estabelecer medidas que, se adotadas, apresentem um prognóstico positivo às organizações. A literatura apresenta uma gama de modelos e frameworks de gestão do conhecimento, como Wiig (1993), Nonaka e Takeuchi (1997), Teixeira Filho (2000), Probst, Raub e Romhardt (2002), Bukowitz e Williams (2002), e Angeloni (2008). Cada um desses modelos é direcionado a um contexto específico. Para esta pesquisa foi utilizado o modelo de Bukowitz e Williams (2002).

\subsection{Modelo de Bukowitz e Williams}

Bukowitz e Williams (2002), em seu Manual de Diagnóstico de Gestão do Conhecimento (DGC), propõem uma metodologia de estruturação dos processos da GC baseada na avaliação de sua situação atual. O DGC disponibiliza ferramentas e técnicas direcionadas para o diagnóstico de cada etapa que compõe o processo de GC em uma organização. O DGC também disponibiliza orientações para a elaboração de um programa de ações para as etapas avaliadas, o que permite a realização de futuras melhorias nos processos de GC na organização.

O diagnóstico de GC estrutura a gestão do conhecimento com base em dois processos: tático e estratégico, os quais são compostos por sete seções (BUKOWITZ; WILLIAMS, 2002) como mostra a Figura 1.

De acordo com o DGC, a estruturação do processo de GC segue do is fluxos de atividades que ocorrem de forma simultânea nas organizaçôes: "(i) a utilização do conhecimento no dia-a-dia para responder às demandas ou oportunidades do mercado e; (ii) o processo, mais a longo prazo, de combinar o intelectual com as exigências estratégicas" (BUKOWITZ; WILLIAMS, 2002, p. 24). O primeiro fluxo (à esquerda na Figura 1) trata do processo tático e se refere ao modo com que as pessoas reúnem as informações necessárias para a realização do seu trabalho diário. Abrange a forma com que essas pessoas utilizam o conhecimento para a geração de valor, como aprendem, como é o meio de criação e desenvolvimento desse conhecimento para o sistema, e para o reuso na resolução de problemas (BUKOWITZ; WILLIAMS, 2002). 0 processo tático é composto por quatro seções denominadas: Obtenha, Utilize, Aprenda e Contribua, as quais são detal hadas a seguir.

Seção 01 - Obtenha: as pessoas e/ou equipes devem encontrar as informações corretas no momento correto, e as organizações devem disponibilizar ferramentas que permitam 0 acesso, 0 gerenciamento e 0 armazenamento de tais informações (BUKOWITZ; WILLIAMS, 2002).

Seção 02 - Utilize: as pessoas e/ou equipes devem buscar constantemente fontes novas e seguras para conseguirem inovar e atender às necessidades dos clientes. Já as organizações devem disponibilizar ambientes e melhores práticas para estimular a criatividade e a utilização da informação (BUKOWITZ; WILLIAMS, 2002). 
Figura 1 - Estruturação dos processos de gestão do conhecimento

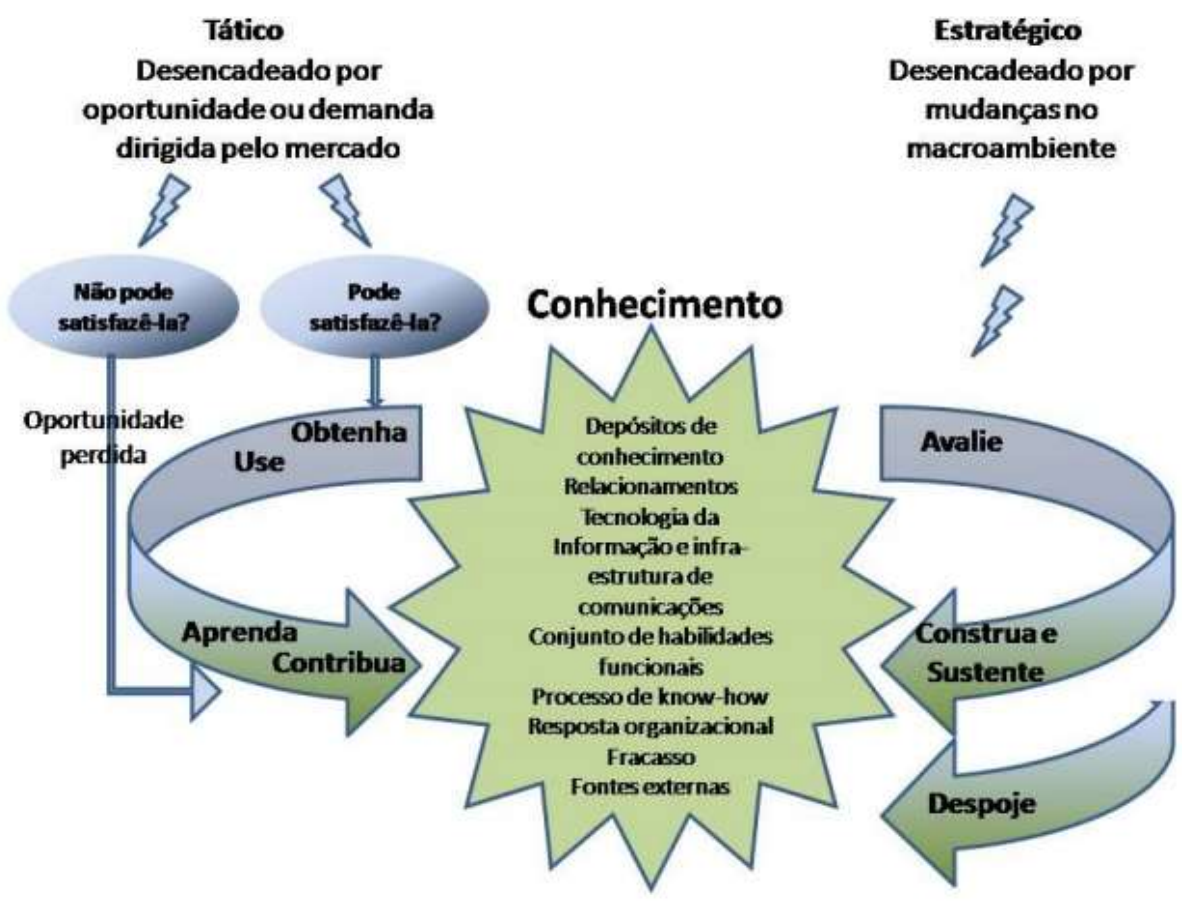

Fonte: Adaptado de Bukowitz e Williams (2002, p. 24).

Seção 03 - Aprenda: etapa do processo de GC que visa integrar novas formas de aprendizagem com as diversas maneiras com que os indivíduos trabalham. É importante que as pessoas e/ou equipes entendam e aprendam com algumas ações que afetam os resultados no trabalho diário, para que consigam melhorar os resultados futuros. Já as organizações têm como responsabilidade proporcionar um ambiente para assim compreender os diversos estilos de aprendizagem (BUKOWITZ; WILLIAMS, 2002).

Seção 04 - Contribua: refere-se à forma de interpretação (mentalidade) das pessoas de que 0 compartilhamento do conhecimento é perda de tempo e que não agrega valor para quem contribuiu, mas sim para quem recebe. As pessoas e/ou equipes precisam anular essa forma de pensar e desenvolver o desejo de colaboração, enquanto as organizações precisam promover estruturas de apoio a uma cultura direcionada para a colaboração de ideias (BUKOWITZ; WILLIAMS, 2002).

O outro fluxo (à direita na Figura 1) é o processo estratégico. Neste, "a gestão do conhecimento, em nível estratégico, exige uma avaliação contínua do capital intelectual existente e uma comparação com necessidades futuras" (BUKOWITZ; WILLIAMS, 2002, p. 26). O processo estratégico se preocupa com o papel de grupos e lideranças específicas e abrange três seções da gestão do conhecimento, as quais são: Avalie, Construa/Mantenha e Despoje/Descarte, detalhadas na sequência (BUKOWITZ; WILLIAMS, 2002).

Seção 05 - Avalie: os indivíduos e/ou equipes devem buscar formas de mensuração, de baixa complexidade, dos ativos intelectuais no processo de planejamento. Já as organizações têm como responsabilidade mapear e avaliar quais desses ativos poderão ser aproveitados em necessidades futuras (BUKOWITZ; WILLIAMS, 2002).

Seção 06 - Construa/Mantenha: as pessoas e/ou equipes devem buscar com frequência novos experimentos com o objetivo de gerar um diferencial competitivo para a organização, para justificar novos e sistemáticos investimentos realizados. Já a organização tem como principal responsabilidade desenvolver e implementar um bom programa de ação no sentido de favorecer a construção de novo conhecimento (BUKOWITZ; WILLIAMS, 2002).

Seção 07 - Despoje/Descarte: os indivíduos e/ou equipes devem desenvolver e adotar algum método que permita analisar os custos da retenção de conhecimento desnecessário. Para a organização, sua principal responsabilidade é reconhecer que pode existir conhecimento desnecessário e é fundamental incentivar o conhecimento novo (BUKOWITZ; WILLIAMS, 2002). 
A seguir são apresentados os procedimentos metodológicos utilizados para a realização desta pesquisa, a aplicação do modelo de diagnóstico de gestão do conhecimento e, a apresentação e análise dos resultados encontrados.

\section{PROCEDIMENTOS METODOLÓGICOS}

Quanto a sua natureza, essa pesquisa classifica-se como aplicada, ao produzir resultados que podem ser utilizados imediatamente. No que se refere a sua abordagem é qualitativa, por interpretar e atribuir significado aos fenômenos e, quanto aos seus objetivos é descritiva, pois é uma pesquisa que visa descrever fenômenos (MARCONI; LAKATOS, 2003). 0 procedimento técnico utilizado caracteriza-se como caso de estudo baseado na aplicação do modelo de Bukowitz e Williams (2002).

A pesquisa foi realizada com cinco dirigentes de uma organização de pesquisa e desenvolvimento intensiva em conhecimento, localizada em Florianópolis, Santa Catarina. As organizações intensivas em conhecimento são denominadas como tal por desenvolverem produtos e serviços por meio do uso do conhecimento. As organizações de consultoria, P\&D e tecnologia da informação são exemplos de organizações intensivas em conhecimento. Apesar de grande parte das organizações utilizarem conhecimento, o que as diferem é a intensidade com que esse ativo é utilizado (ALVESSON, 2004).

A coleta dos dados ocorreu em maio de 2016 e foi utilizado o instrumento proposto por Bukowitz e Williams (2002). As seções de GC, a quantidade de itens por seção e os critérios de avaliação são apresentados no Quadro 1. De acordo com o modelo de DGC, cada seção pode receber a pontuação máxima de 60 pontos (20 afirmativas x peso 3). A pontuação máxima possível do DGC é 420 (60 pontos x 7 seções) (BUKOWITZ; WILLIAMS, 2002).

Quadro 1 - Seções e critérios de avaliação da gestão do conhecimento

\begin{tabular}{|c|c|c|}
\hline Seções de GC & Itens por seção & Critério de avaliação \\
\hline Obtenha & 20 afirmativas & \multirow{7}{*}{$\begin{array}{l}\text { Forte (a afirmativa é fortemente descritiva na minha organização) - } \\
\text { Peso } 3 \\
\text { Moderado (a afirmativa é moderadamente descritiva na minha } \\
\text { organização) - Peso } 2 \\
\text { Fraco (a afirmativa é fortemente descritiva na minha organização) - } \\
\text { Peso } 1\end{array}$} \\
\hline Utilize & 20 afirmativas & \\
\hline Aprenda & 20 afirmativas & \\
\hline Contribua/Avalie & 20 afirmativas & \\
\hline $\begin{array}{l}\text { Construa/Manten } \\
\text { ha }\end{array}$ & 20 afirmativas & \\
\hline Despoje/Descarte & 20 afirmativas & \\
\hline Total & 120 afirmativas & \\
\hline
\end{tabular}

Fonte: Adaptado de Bukowitz e Williams (2002).

O tratamento dos dados baseou-se na metodologia proposta por Bukowitz e Williams (2002), que consiste no cálculo de frequência absoluta, ponderação e dados percentuais, os quais foram executados por meio de uma planilha eletrônica. A partir desse procedimento os resultados gerados foram analisados e discutidos de forma descritiva.

\section{APRESENTAÇÃO E DISCUSSÃO DOS RESULTADOS}

O resultado alcançado nas seções e a média geral do diagnóstico de gestão do conhecimento (Figura 2) demonstram que a organização pesquisada se enquadra no intervalo de pontuação de $30 \%$ a $70 \%$, considerado aceitável por Bukowitz e Williams (2002). 0 teste de campo realizado por Bukowitz e Williams (2002) demonstrou que as organizações participantes apresentaram média geral igual a 55\% no diagnóstico da gestão do conhecimento, já a organização de $P \& D$ pesquisada apresentou média igual a $58 \%$. Comparativamente a outras pesquisas anteriores, a média geral obtida (58\%) está acima do máximo anterior (55\%). 
Figura 2 - Resultado (em percentual) de cada seção do DGC e a média geral

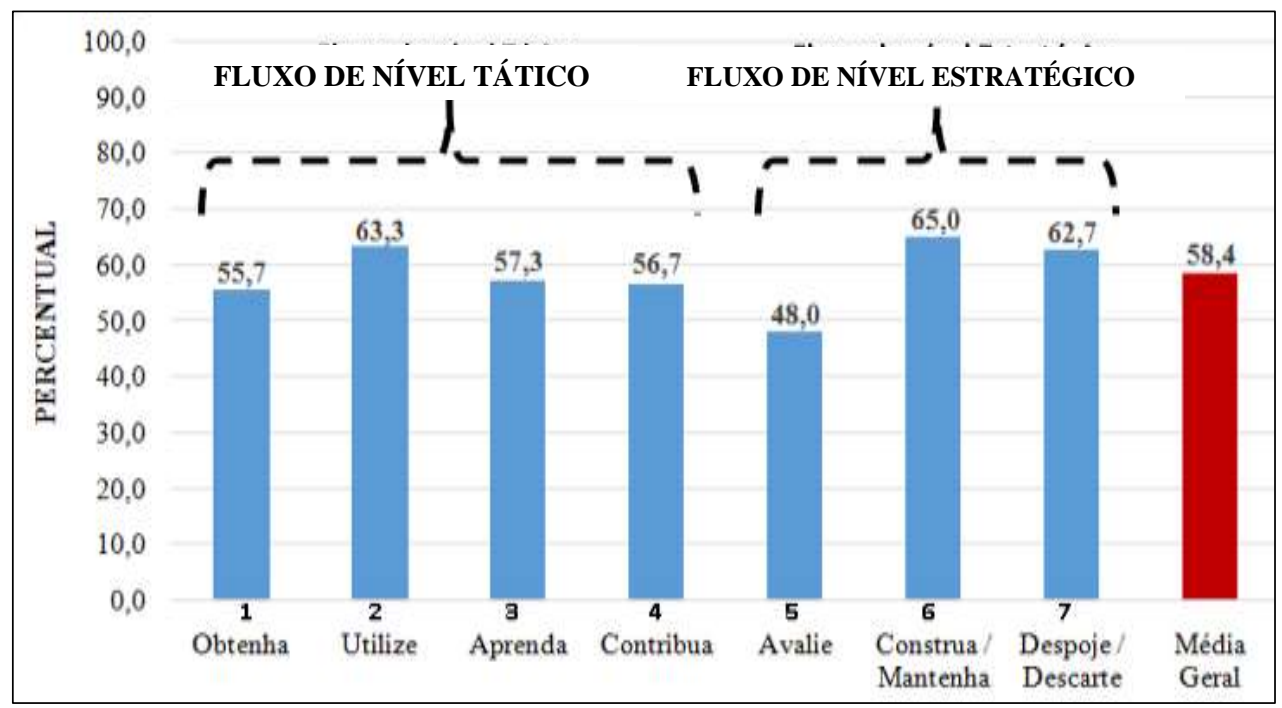

Fonte: Dados da pesquisa (2016)

Os resultados indicam a necessidade de a organização pesquisada aprimorar seu processo de GC. A média geral é decorrente dos resultados parciais em cada seção e indica quais aspectos podem ser aperfeiçoados. Destacam-se os processos Utilize, Construa e Mantenha, Despoje e Descarte, que apresentaram avaliação acima de $62 \%$, o que sugere maior atuação e propriedade dos resultados.

Analisando mais especificamente os resultados, na Seção 01-Obtenha, a avaliação dos dirigentes demonstrou uma maior facilidade na identificação e acesso aos especialistas, sendo que seu domínio de conhecimento e atuação são de fácil identificação. As demais afirmativas dessa seção apresentaram avaliação média ou fraca. Como ponto negativo, destaca-se 0 aspecto de que as pessoas podem ter dificuldades para tornar rotineiro o seu ambiente informacional. Embora a atualização dos repositórios de conhecimento seja classificada como "normal", as tarefas de pesquisa informacional e as bases de dados a serem pesquisadas ficam abaixo do resultado esperado, conforme o DGC. Outro aspecto considerado fraco, na percepção dos dirigentes, consiste nos especialistas em informação da organização ajudarem pouco as pessoas a utilizarem instrumentos online (inclusive a internet).

"A documentação em um sistema de gestão adquire a importante função de assegurar que haja um mínimo de ordem no modo como a organização conduz seus negócios" (COSTAL; MARTINS, 2011, p. 4). Os dirigentes pesquisados demonstraram que não é feita de forma efetiva a distinção entre a informação que deveria ser controlada centralmente e àquela que todos da organização deveriam ser livres para documentar e compartilhar.

Na Seção 02 - Utilize, que se enquadra em um patamar acima da média, a partir da avaliação dos dirigentes. Nessa seção, a flexibilidade do ambiente se destaca positivamente, pois permite a todos executarem as tarefas com menor esforço. Outros aspectos bem avaliados se referem ao fato de que a hierarquia não é um empecilho e que todos têm a oportunidade de descrever como as suas decisões afetam o desempenho geral da organização. Os aspectos negativos identificados consistem na existência de fracos protocolos de segurança sobre confidencialidade e os aspectos financeiros da organização, que não são compreendidos por toda a organização.

A respeito da Seção 03 - Aprenda, seu ponto forte identificado na organização pesquisada consiste na discordância de pontos de vista, de modo que a curiosidade e as responsabilidades são aspectos de valor para o aprendizado organizacional. A utilização de jogos e simulações como alternativa para fazer as pessoas refletirem sobre as situações de negócios não são frequentes na organização pesquisada. Ao final dos projetos, os dirigentes indicaram que não há uma avaliação efetiva do desempenho, o que permitiria a identificação dos erros cometidos e seu melhoramento. Esse resultado pode ser decorrente da escassez de tempo.

Na Seção 04 - Contribua, a ausência de incentivos ou benefícios ao compartilhamento de conhecimento atua negativamente na organização, embora exista o reconhecimento de autoria de cada 
contribuição. Outro ponto negativo consiste no fato de os gerentes ou coordenadores de conhecimento não terem dedicação exclusiva na organização. Em contrapartida, as múltiplas comunidades e as interações físicas entre os participantes são positivas ao facilitar a transferência do conhecimento implícito.

A Seção 05 - Avalie apresentou a avaliação mais baixa. Como fatores positivos destacam-se que há um reconhecimento de que o conhecimento é o principal fator que gera valor para a organização e que sua gestão vem sendo praticada há algum tempo, mesmo sem o seu reconhecimento formal. Em contrapartida, identificaram-se elementos considerados negativos para a organização, como a ausência de métricas nos diversos estágios do processo e nos resultados e; a ausência de publicações formais da organização, direcionadas ao público interno e externo, que relatam a qualidade e o nível em que o conhecimento é gerido.

A Seção 06- Contribua/Mantenha apresentou a melhor avaliação, segundo a percepção dos dirigentes pesquisados, que indicaram os seguintes pontos a serem melhorados: incluir explicitamente os clientes nos processos e definir explicitamente os profissionais com o papel de líder no processo de GC da organização e; classificar as informações quanto a sua confidencialidade das informações. Como elemento positivo identificou-se que o uso de tecnologias (independentemente da sua autoria) pode ser utilizado por todos, e que a GC deve ser preocupação de todos, não apenas dos gerentes. A contribuição das tecnologias da informação e comunicação (TICS) é considerada adequada e confiável e, a cada dia tem-se buscado novas alianças estratégicas e parcerias.

Finalmente, a Seção 07- Despoje/Descarte apresentou uma avaliação acima da média também. Destacam-se como pontos negativos: a inexistência de um plano que estabeleça o descarte, especialmente porque não há regras baseadas em estratégias do capital intelectual e nas projeções financeiras e; a inexistência de um plano para que os membros da organização adquiram outras expertises por meio da sua atuação em outras organizações. Como pontos negativos destacam os seguintes: os gestores apresentam plena participação em grupos de pesquisa e; o fato de os gestores terem um olhar voltado às competências dos seus profissionais antes da escolha de um novo colaborador e antes de demitir algum de seus profissionais, no sentido de identificar se as habilidades e expertises deles podem ser utilizadas em outra função/atividade na organização.

A avaliação total dos resultados é apresentada de forma detalhada na Tabela 1. Grande parte das seções apresentou pontuação média, o que influenciou no resultado final $(245,2)$. Isso indica que, apesar de a empresa atuar no setor de $P \& D$, caracterizado como intensivo em conhecimento, os resultados mostram que a GC na organização apresenta elementos de processos da GC que necessitam de melhorias, conforme a percepção dos dirigentes pesquisados.

Tabela 1 - Resultado por seção do DGC na organização pesquisada

\begin{tabular}{clc|} 
Fluxo de atividades & Seção do DGC & $\begin{array}{c}\text { Pontuação por seção de } \\
\text { GC }\end{array}$ \\
\hline \multirow{3}{*}{ Tático } & 1 - Obtenha & 33,4 \\
& 2 - Utilize & 38,0 \\
& 3 - Aprenda & 34,4 \\
& 4 - Contribua & 34,0 \\
& 5 - Avalie & 28,8 \\
\hline \multirow{2}{*}{ Estratégico } & 6 - Construa/Mantenha & 39,0 \\
& 7 - Despoje/Descarte & 37,6 \\
& Total & $\mathbf{2 4 5 , 2}$
\end{tabular}

No DGC a pontuação máxima a ser encontrada é 420 pontos com máxima de 210 pontos, sendo que a pontuação obtida na organização em estudo foi de 245,2 pontos. Por meio da Tabela 1 identificam-se as áreas que podem ser melhoradas, o que gera implicações no crescimento geral dos processos de GC, com destaque para a Seção 05 Avalie, que representou o menor resultado. 
A estratégia de GC envolve uma etapa de avaliação que desempenha a importante função de monitorar o progresso e a realização bem-sucedida dos objetivos e iniciativas desse processo (DALKIR, 2005). 0 processo tático aborda as etapas básicas para a GC e requer o envolvimento de todos na organização em algum grau (BUKOWITZ; WILLIAMS, 2002). As seções que apresentaram as menores pontuações pertencem a esse processo de GC. 0 envolvimento possibilita que os funcionários compartilhem suas especialidades entre si, o que pode resultar em profissionais mais comprometidos para utilizar seus conhecimentos para a entrega de melhores resultados em seus trabalhos (CHONG; $\mathrm{CHOI}, 2005$ ).

A partir do resultado geral obtido foi elaborado um programa de ações, direcionadas para possíveis melhorias nas seções do processo de GC com menor avaliação na organização pesquisada. Com base na metodologia proposta por Bukowitz e Williams (2002), cada ação apresenta o problema identificado, a responsabilidade da organização para a resolução de tal problema e as ações específicas de melhorias, subdivididas em imperativos (abordagens). 0 plano de ações (Quadro 2) aborda os processos de GC tanto do fluxo tácito quanto do estratégico. As ações de melho rias são voltadas para as seções que contribuíram para a redução da média dos resultados (58,4\%). Assim, foram desconsideradas do plano as seções 02 (Utilize), 06 (Construa/Mantenha) e 07 (Despoje/Descarte) por apresentarem avaliação acima da média.

Quadro 2 - Ações recomendadas para as seções abaixo da média geral da empresa

\section{Seção Ações recomendadas}

1. Desenvolver linhas de orientação breves que ajudarão a enquadrar as solicitações de informação para torná-las mais eficientes;

2. Com a ajuda dos líderes, coletar informação sobre a especialização e as competências das Seção 01 pessoas;

Obtenha 3. Reunir uma equipe de gestão de informação para determinar qual informação necessita ser

$(55,7 \%) \quad$ "empurrada" e qual pode residir em uma intranet ou em bases de dados para os usuários "puxarem" quando necessário;

4. Reunir grupos de pessoas em papéis da área do conhecimento para trocar ideias e melhores práticas;

5. Examinar partes de informação que estão alojadas em vários locais (repositórios e intranet, por exemplo), conferindo a atualidade e a redundância.

6. Examinar os gerentes operacionais e funcionais e/ou de processo para verificar até onde eles acreditam que é importante que todos na organização entendam a ligação entre estratégia e

Seção 03 as suas tarefas individuais ou de equipe;

Aprenda 7. Discutir as maneiras com as quais o desempenho deveria ser melhorado e o lapso de tempo

$(57,3 \%) \quad$ até que esse melhoramento seja visível;

8. Identificar um gerente que tenha interesse e trabalhar com ele, para fazer mudanças pequenas no processo de trabalho, de modo que os erros sejam mais visíveis e as discussões sobre a situação da empresa. Monitorar o desempenho ao longo do tempo para determinar se, quando e como ele muda.

9. Identificar situações que tem impacto negativo sobre o compartilhamento de conhecimento;

10. Avaliar os custos e os benefícios de remover as barreiras do compartilhamento do conhecimento;

11. Discutir maneiras de compensar as pessoas, por compartilharem conhecimento que sejam mais efetivas na cultura da organização. Definir os termos de contribuição necessários para

Seção 04 obter os benefícios;

Contribua 12. Informalmente, entrevistar membros da organização, para determinar se acreditam que existe

$(56,7 \%) \quad$ um contrato de reciprocidade entre eles e a organização e entre eles e os outros membros;

13. Examinar os sistemas groupware, em busca de oportunidades para melhorar a atribuição e o feedback àqueles que contribuem;

14. Entrevistar aqueles que contribuem frequentemente com os sistemas grupais, assim como aqueles que raramente o fazem, para determinar se o retorno ou a melhoria da atribuição poderiam estimular a contribuição.

15. Definir os tipos fundamentais de capital humano, organizacional e de cliente que a organização deveria medir e monitorar; 


\begin{tabular}{|c|c|}
\hline $\begin{array}{c}\text { Seção } 05 \\
\text { Avalie } \\
(48 \%)\end{array}$ & $\begin{array}{l}\text { 16. Iniciar uma série de diálogos pela organização para aprimorar a ideia inicial sobre o capital } \\
\text { intelectual. Engajar os principais clientes e fornecedores nas discussões para ampliar a } \\
\text { perspectiva sobre as formas de capital intelectual que devem ser monitoradas e medidas; } \\
\text { 17. Investigar os níveis atuais de entendimento das pessoas da organização para determinar em } \\
\text { que medida a organização deve ser educada sobre a gestão do conhecimento; } \\
\text { 18. Identificar os principais capacitores que apoiam o processo de gestão do conhecimento; } \\
\text { 19. Verificar a intensidade do atual ambiente da empresa de avaliação pela identificação de } \\
\text { quantos tipos de informação são rastreados rotineiramente hoje; } \\
\text { 20. Identificar as principais barreiras para criar e distribuir relatórios sobre o conhecimento; } \\
\text { 21. Fazer parcerias com grupos de stakeholders importantes, para criar protótipos de processos } \\
\text { de relatório e esclarecer os benefícios de compartilhar informacão. }\end{array}$ \\
\hline
\end{tabular}

Fonte: Adaptado de Bukowitz e Williams (2002, p. 363-366).

O programa de ações propõe uma série de desafios a serem melhor trabalhados pela organização pesquisada como forma de atingir um nível de excelência na geração, tratamento, contribuição e renovação do conhecimento, proporcionando-Ihe melhor utilização dos seus recursos tangíveis e intangíveis.

\section{CONSIDERAÇÕES FINAIS}

Tendo em vista a importância da GC no contexto atual em que as organizações estão inseridas, essa pesquisa realizou um diagnóstico de gestão do conhecimento em uma organização de pesquisa e desenvolvimento, intensiva em conhecimento. 0 estudo demonstrou que a organização obteve avaliação acima da média para a gestão dos conhecimentos existentes em seu ambiente. Por outro lado, os resultados indicam a necessidade de melhorias em alguns processos de GC. Essas melhorias tratam dos processos de GC pertencentes principalmente ao nível tático da organização.

A respeito do nível estratégico, as seções melhores avaliadas pelos dirigentes foram Construa/Mantenha e Despoje/Descarte. No entanto, a seção com a menor avaliação se refere à Seção 05 (Avalie), em que o planejamento e o mapeamento de valores necessitam ser aperfeiçoados. No que se refere ao nível tático, apenas a Seção Utilize apresentou maior avaliação dos dirigentes. As Seções 01 (Obtenha), 03 (Aprenda) e 04 (Contribua) apresentaram a avaliação mais baixa, e contribuíram consideravelmente para a redução da média da GC na organização pesquisada.

Essa pesquisa traz implicações práticas sobre a possibilidade de avaliação dos processos de gestão do conhecimento na organização pesquisada. Os resultados possibilitam visualizar os processos que necessitam de melhorias e aqueles que apresentaram boa avaliação de seus processos, a partir da percepção dos dirigentes pesquisados. As melhorias consideradas necessárias e indicadas nessa pesquisa podem ocorrer a partir do plano de ações apresentado na seção anterior. 0 plano de ações pode auxiliar a organização no processo de efetividade da gestão de seus conhecimentos.

Com base nos resultados encontrados sugere-se como pesquisas futuras aplicar 0 Diagnóstico de Gestão do Conhecimento na organização pesquisada envolvendo todos os seus profissionais, para comparar os resultados das percepções da GC entre os diferentes níveis hierárquicos sobre o fluxo tático e estratégico da GC. Recomenda-se também, reaplicar o DGC para analisar se os processos de GC com baixa avaliação, principalmente, 
apresentaram melhorias ao longo do tempo, tendo em vista que foi proposto um plano de ações. Finalmente, outra possibilidade de pesquisa futura consiste na realização de estudo sobre a Gestão do Conhecimento sob o prisma do Modelo de Bukowitz e Williams (2002) e os aspectos modernos da Governança de Conhecimento, tema este que emerge especialmente baseado nos conceitos de Foss (2007).

\section{REFERÊNCIAS}

ALVESSON, M. Knowledge work and knowledge - intensive firms. Oxford: Oxford University Press, 2004.

BRITO, L. M. P.; OLIVEIRA, P. W. S.; CASTRO, A. B. C. Gestão do conhecimento numa instituição pública de assistência técnica e extensão rural do Nordeste do Brasil. Revista de Administração Pública, Rio de Janeiro, v. 46, n. 5, p. 1341-1366, 2012.

BUKOWITZ, W. R.; WILLIAMS, R. L. Manual de gestão do conhecimento: ferramentas e técnicas que criam valor para a empresa. Porto Alegre: Bookman, 2002.

COSTAL, G. C. S. Z; MARTINS, R. A. Dificuldades na informatização de um sistema de gestão da qualidade baseado na norma ABNT ISO 9001. In: ENCONTRO NACIONAL DE ENGENHARIA DE PRODUÇÃO, 31., 2011, Belo Horizonte. Anais [...]. Belo Horizonte: ABEPRO, 2011.

CHONG, S. C.; CHOI, Y. S. Critical Factors In The Successful Implementation Of Knowledge Management. Journal of Knowledge Management Practice, [S.I.], v. 6, [não paginado], 2005.

DALKIR, K. Knowledge management in theory and practice. Boston: Elsevier, 2005.

DAVENPORT, T. H.; PRUSAK, L. Conhecimento empresarial: como as organizações gerenciam o seu capital intelectual. Rio de Janeiro: Campus, 1998.

FLEURY, M. T. L.; OLIVEIRA J R., M. M. (Org.). Gestão estratégica do conhecimento: integrando aprendizagem, conhecimento e competências. São Paulo: Atlas, 2001.

FOSS, N. J. New Organizational forms: critical perspectives. International J ournal of the Economics of Business, [S.I.], v. 9, n. 1, p. 1-8, 2002.

The Emerging Knowledge Governance Approach: challenges and characteristics. Organization, London, v. 14 n. 1, p. 29-52, 2007.

GARCIA, O. P. G.; COLTRE, S. M. Knowledge management as a determining factor in the retention of professionals in the industry: a case study in an organization in the furniture industry. Brazilian Business Review (Portuguese Edition), Vitória, v. 14, n. 2, p. 182-203, 2017.

JANUZZI, C. S. C.; FALSARELLA, O. M.; SUGAHARA, C. R. Gestão do conhecimento: um estudo de modelos e sua relação com a inovação nas organizações. Perspectivas em Ciência da Informação, Belo Horizonte, v. 21, n. 1, p. 97-118, jan./mar. 2016.

LANGLOIS, R. N. The vanishing hand: the changing dynamics of industrial capitalism. Industrial and corporatechange, [S.I.], v. 12, n. 2, p. 351-385, 2003.

MADHOK, A. Crossroads - the organization of economic activity: transaction costs, firm capabilities, and the nature of governance. Organization Science, [S.I.], v. 7, n. 5, p. 577-590, 1996.

MARCONI, M. A.; LAKATOS, E. M. Fundamentos de metodologia científica. 5. ed. São Paulo: Atlas 2003. 
NADAI, F. C. Uma análise crítica do termo "organizações intensivas em conhecimento". Revista Gepros Gestão de Produção, Operações e Sistemas, Bauru, SP, v. 2, n. 3, p. 97-106, 2006.

NONAKA, I.; TAKEUCHI, H. Criação de conhecimento na empresa: como as empresas japonesas geram a dinâmica da inovação. Rio de J aneiro: Campus, 1997.

OLIVA, F. L. Knowledge management barriers, practices and maturity model. Journal of Knowledge Management, [S.I.], v. 18, n. 6, p. 1053-1074, 2014.

SCHOLL, W. et al. The future of knowledge management: an international Delphi study. Journal of knowledge management, [S.I.], v. 8, n. 2, p. 19-35, 2004.

SOUZA, A. M.; KURTZ, D. J. Análise de modelos para a Gestão do conhecimento organizacional: o caso serviço social da indústria-SESI/PE. International J ournal of Knowledge Engineering and Management (IJ KEM), Florianópolis, v. 3, n. 6, p. 64-88, 2014.

VASCONCELOS, A. C. F.; CÂNDIDO, G. A. Diagnóstico de gestão do conhecimento como mecanismo para criação de valor: um estudo exploratório no SEBRAE-PB. In: SIMPÓSIO DE GESTÃO DA INOVAÇÃO TECNOLÓGICA, 25., Brasília, 2008. Anais [...]. Brasília: SEBRAE-PB, 2008. 